\title{
Nikolaus Poppe 1897-1991
}

Nikolaus (Nikolaj Nikolajevix) Poppe, Altmeister der Altaistik und Ehrenmitglied der Finnisch-Ugrischen Gesellschaft, ist am 8. Juni 1991 in Seattle verstorben. Bei seinem Hinscheiden hatte er das ehrwürdige Alter von fast 94 Jahren erreicht. Man hat das Gefühl, als sei eine ganze Epoche mit ihm zu Ende gegangen, die Epoche grundlegender Feldforschung und souveräner Beherrschung aller Bereiche der vergleichenden altaischen Sprachwissenschaft.

Nikolaus Poppe wurde am 8. August 1897 in der Hafenstadt Chefoo (heute Yantai) auf der Shandonger Halbinsel südöstlich von Beijing geboren. Sein Vater Nikolaus Edwin Poppe arbeitete als Sekretär am Kaiserlichen Russischen Konsulat in Tientsin (Tianjin) - die Qualifikation für seine Stellung hatte er durch Studien des Chinesischen, Mandschurischen und Mongolischen sowie der ostasiatischen Geschichte und anderer orientalischer Disziplinen an der St. Petersburger Universität erworben.

Chinesisch war die erste Sprache, die der kleine Nikolaj Nikolajevix erlernte; auch im Gespräch mit seiner Mutter benutzte er sie. Die drohenden Ereignisse des Boxeraufstandes verursachten, daß Mutter und Sohn 1901 zur Großmutter mütterlicherseits nach St. Petersburg geschickt wurden. Der Junge hatte es nicht leicht: Alles war fremd, das Klima rauh, er vermißte China und die Chinesen sehr. Er sprach erst nur Chinesisch, lernte aber bald auch Russisch. In seinen Erinnerungen erzählt er, daß er sich in St. Petersburg nie richtig zu Hause fühlte und immer anderswohin ins Ausland fahren wollte. Er sagt auch, daß dies wahrscheinlich die Ursache war, warum er später orientalische Sprachen zu studieren beschloß und als Konsulatsangestellter nach China oder in irgendein anderes fernöstliches Land gehen wollte. Unter solchen Umständen entdeckte er seinen Wunsch, ein Gelehrter zu werden.

Im Jahre 1903 wurde der Vater als russischer Konsul nach Tsitsihar in der nördlichen chinesischen Provinz Heilongjiang gesandt, und die ganze Familie versammelte sich dort. Vorüberziehende mongolische Karawanen machten einen tiefen Eindruck auf den kleinen Nikolaus; er sagt, dies könne ein Grund für seine spätere intensive Beschäftigung mit der Mongolistik sein. Der Russisch-japanische Krieg 1904-05 setzte dem Aufenthalt in Tsitsihar ein Ende. Mutter und Kinder mußten nach St. Petersburg zurückkehren. Der Vater blieb im Dienst, mit unglücklichen Folgen: Er wurde im Jahre 1913 in Harbin ermordet. Nach diesem Ereignis war es in der Familie ein Tabu, über orientalische Studien zu sprechen. 
Die Familie verbrachte die Sommermonate auf finnischem Boden in Karelien: zunächst in Raivola, dann in Antrea und Parikkala. Diese Tatsache und finnische Schulkameraden bewirkten, daß Nikolaus bald ein Kenner der verschiedenen karelischen Dialekte neben der finnischen Schriftsprache wurde. Einmal kamen ein paar ostkarelische Liedersänger zu Besuch ins Sommerhaus in Parikkala und sangen von Väinämöinens Bootsbau aus dem Kalevala. Auch jenes Ereignis machte einen tiefen Eindruck auf Nikolaus und er bat seine Mutter, ihm ein Exemplar des Kalevala zu besorgen. Die faszinierende Lektüre des finnischen Nationalepos verursachte, da $B$ die Geschichte der Finnen und anderer finnisch-ugrischer Völker sein Hauptinteresse wurde. Später las er Yrjö-Koskinens Geschichte Finnlands und Castréns Reisebeschreibung aus Sibirien sowie dessen ethnologische Vorlesungen über altaische Völkerschaften. Er beschloß, die Sprache und Volksdichtung der Finnen und anderer Völker, Mongolen und Türken einberechnet, die Castrén als sprachlich verwandt betrachtete, zu studieren.

In der Schule der Reformierten Gemeinden in St. Petersburg lernte Nikolaus Deutsch, Französisch, Russisch, Latein und Griechisch. Der Lehrer im Griechischen, Dr. Erich von Voss, der über Sanskrit disputiert hatte, lehrte den Jungen eine formanalytische Übersetzungsmethode und andere nützliche philologische Einsichten. Im Jahre 1916 legte er die Reifeprüfung mit Auszeichnung ab. Die finnisch-ugrische und altaische Linguistik und Volksdichtung lockten ihn, aber unsichere Berufsaussichten auf diesem Gebiet und das durch die Ermordung des Vaters verursachte traumatische Verhältnis der Familie zu orientalischen Studien im allgemeinen und speziell zu allem, was mit dem Fernen Osten zu tun hatte, bewirkten, daß er an der Petrograder Universität mit Naturwissenschaften und Medizin begann.

Nach einem kurzen Dienst im alten Regiment von Semjonovskij 1917-18 nahm Nikolaus Poppe seine Universitätsstudien wieder auf, diesmal jedoch in einer völlig anderen Richtung; ungeachtet der schlechten Aussichten wollte er sich nun den orientalischen Studien widmen. Finnougristik konnte man damals nur in Helsinki oder Budapest studieren, aber die Revolution hatte die Grenze zwischen Rußland und Finnland endgültig geschlossen. Auf Anraten von Andrej Rudnev, Professor der mongolischen Literatur, beschloß der junge Kolja, als Hauptfach weiter Mongolistik zu studieren. Dies war der eigentliche Beginn einer glänzenden Karriere, die zu reichen Ergebnissen führte.

Da Rudnev Sowjetrußland verlassen hatte, wurde Nikolaus Poppe ein Schüler des vielseitigen Gelehrten Boris Vladimircov, der jedoch versuchte, die wissenschaftlichen Fortschritte seines begabten Schülers zu erschweren. Unter Władysław Kotwicz studierte Poppe Mandschurisch und Oiratisch/Kalmückisch, unter A. N. Samojlovið türkische Sprachen. Sein erstes Buch, eine jakutische Grammatik (gedruckt i.J. 1926), schrieb er auf Anraten von Samojlovic. Seine sonstigen Lehrer der Turkologie waren S. E. Malov (Uigurisch) 
und P. A. Falev (Osmanisch, Nogajisch und Kasachisch). Tibetisch studierte er bei F. I. Scerbackoj und die Geschichte der Orientalistik bei V. V. Bartol'd. Als seine Lehrer nennt Poppe u.a. auch L. V. Scerba und N. J. Marr (Linguistik) und L. J. Sternberg (Ethnographie).

Im Jahre 1919 verschaffte Professor Sternberg Poppe eine Stellung als Forschungsassistent beim Geographischen Institut, in der Abteilung für Ethnographie. Seine erste Aufgabe war eine ethnographische Karte der finnischen Bevölkerung des Petrograder Distrikts zusammenzustellen; 1920 wurde eine Expedition zu den Kareliem von Tver unternommen. In demselben Jahr begann Poppe als Lehrer der Mongolistik an dem kürzlich gegründeten Institut für lebende orientalische Sprachen. Daneben unterrichtete er mongolische und kalmückische Studenten im Russischen, in der Mathematik und in anderen Fächern.

Nachdem Poppe im Februar 1921 die Universität absolviert hatte, wurde er im Sommer mit Kalmücken nach Astrachan gesandt, wo er erkrankte und sogar an Tuberkulose litt. Er wurde auch Assistent am Asiatischen Museum der Akademie der Wissenschaften; seine Aufgabe war es, die unzähligen mongolischen Handschriften und Xylographdrucke zu katalogisieren und zu beschreiben. Im gleichen Jahr erhielt er die Stellung eines Dozenten des Mongolischen. 1919-23 wirkte er bei der toponymischen Kommission mit. Er sollte die Hydronymie am Ilmensee, hauptsächlich jene finnischen Ursprungs, erforschen.

Sein Magisterexamen legte Poppe i.J. 1923 ab. Später im gleichen Jahr heiratete er Natalija Belolipskaja; sie bekamen bald zwei Söhne, Valerian und Nikolaj. Den ersten internationalen Kongreß erlebte Poppe in Baku, wo sich 1926 die größten Turkologen jener Zeit versammelten. Seine große Hoffnung, die Mongolen in ihrer Heimat erforschen zu können, erfüllte sich kurz danach, indem er mit einer Expedition nach Burjatien und in die Mongolei gelangte. Er besuchte interessante Denkmäler und sammelte sprachliche sowie volkskundliche Materialien. Poppe sprach schon damals mühelos Mongolisch, da er es in Leningrad mit seinem Schüler Gombodschab studiert hatte. 1927 arbeitete er erneut in der Mongolei, mit großem Erfolg. Er konnte nämlich das Dagurische und einige Dialekte gründlich erforschen. Diese jährlichen Besuche endeten bereits 1929, da er kein Auslandsvisum mehr erhielt; in Burjatien konnte er jedoch bis 1941 weiterarbeiten. Seit 1925 war Nikolaus Poppe Ordinarius der Leningrader Universität gewesen; im Jahre 1928 erhielt er die gleiche Stellung am Institut für lebende orientalische Sprachen. Als der Doktorgrad in der Sowjetunion wieder eingeführt wurde, wurde er i.J. 1934 auch Nikolaus Poppe verliehen.

Die paranoiden Jahre des großen Terrors setzten dem Schaffen vieler brillanter Forscher ein Ende. Rund 40 der 90 Mitglieder des Orientalischen Instituts wurden arrestiert. Die Zensur forderte absurde Umgestaltungen sogar in rein wissenschaftlichen Texten. Die dreißiger Jahre bedeuteten eine beinahe völlige Isolierung für sowjetische Wissenschaftler. All dies mußte auch Poppe durchmachen. 
Wegen des Krieges wurde die Akademie der Wissenschaften i.J. 1941 evakuiert; die Forscher wurden an Ortschaften geschickt, die ihrer Spezialisierung entsprachen. Als Aufenthalt für Nikolaus Poppe wurde die kalmückische Stadt Elista gewählt. Auch seine Familie konnte er vom nahen Kaukasien dorthin kommen lassen. Im darauffolgenden Frühjahr erhielt er eine Lehrerstellung am Pädagogischen Institut des kaukasischen Distrikts Karatschai, wo die Familie häufig ihre Sommerferien verbracht hatte. Die deutschen Truppen rückten näher; plötzlich war der ganze Ort okkupiert. Er befand sich jetzt hinter der Front und es war ihm, dem Gelehrten mit zahlreichen deutschen Ahnen, nun möglich, mit seiner Familie nach Berlin zu übersiedeln.

Nach schwierigen Jahren wurde Nikolaus Poppe i.J. 1949 an die Universität Washington in Seattle berufen. Als Professor hielt er Vorlesungen hauptsächlich in seinem Lieblingsfach, in der Mongolistik. Viele seiner ersten Studenten wurden später berühmt, u. a. John R. Krueger, James E. Bosson, Robert A. Rupen, David Farquhar, Pao Kuo-yi und Hidehiro Okada. Seine in Leningrad gebliebene wissenschaftliche Bibliothek wurde allmählich neu aufgebaut, zum Teil durch freundliche Geschenke von Kollegen. Poppes erste Frau Natalija war 1949 gestorben; 1952 heiratete er Edith Ziegler, eine Jugendbekanntschaft aus St. Petersburg.

Nikolaus Poppe wurde i.J. 1968 emeritiert; kurz danach wurde seine Disziplin an der Universität Washington aufgelöst. Dies und andere damit verknüpfte Tatsachen deprimierten ihn sehr. Aber seine wissenschaftliche Tätigkeit florierte weiter. An der altaistischen Diskussion nahm er eifrig teil. Obwohl sein gesamtes Schaffen es durchaus gestattet, ihn als einen Linguisten, Philologen, Literarhistoriker, Ethnologen und Historiker zu bezeichnen, wollte er selbst den Begriff "Altaistik" sehr eng definieren; seiner Meinung nach ist Altaistik die deskriptive, historische und vergleichende Untersuchung der sogenannten altaischen Sprachen, einschließlich der Dialektologie. Dagegen gehören Literatur oder Geschichte derselben Völker nicht dazu, weil jene Völker keine kulturelle oder historische Einheit bilden. Dadurch ist Altaistik ein Zweig der Linguistik. Zentrales Thema der Altaistik ist die Verwandtschaftstheorie der türkischen, mongolischen und mandschu-tungusischen Sprachen. Poppe war von deren genetischem Verhältnis fest überzeugt und betrachtete sich darin als einen Schüler Ramstedts. Positiv stand er auch den Versuchen gegenüber, Koreanisch und Japanisch als Glieder einer proto-nordasiatischen Urgemeinschaft nachzuweisen, wovon das Proto-Altaische den einen Zweig bilden würde.

Poppes literarische Tätigkeit ist überwältigend. Schon in den Jahren 1924-77 veröffentlichte er etwa 50 Monographien (Neudrucke inbegriffen), 210 Aufsätze und 170 Rezensionen. Darunter zu erwähnen sind seine Grammatiken: des Jakutischen (1926), der mongolischen Schriftsprache (russ. 1937, engl. 1954), des Burjatischen (russ. 1938, engl. 1960), des Khalkha-Mongolischen (1951), des Tatarischen (1963) und des Baschkirischen (1964). Weiter 
sein Lehrbuch des Mongolischen (1932 u. 1940), seine Übersicht der burjatischen Linguistik (1933) und der Quadratschrift (russ. 1941, engl. 1957), sowie seine berühmten Handbücher: Introduction to Mongolian Comparative Studies (1955), Vergleichende Grammatik der altaischen Sprachen I (1960), Introduction to Altaic Linguistics (1965) und Mongolian Language Handbook (1970). Sprachmaterialien, besonders Volksdichtung, befinden sich in vielen hochklassigen Ausgaben: bargusin-tungusische Materialien (1927), dagurische und alarische Dialekttexte (1930-31), solonische Materialien (1931), burjatische Kolchosdichtung (1934) und mongolische Volksdichtung (1955). Die Übersicht der khalkha-mongolischen Heldendichtung (1937) zeigte sein Interesse für Epik; später erwies er den Folkloristen einen großartigen Dienst durch die Übersetzung zahlreicher mongolischer Epen (7 Bände 1975-80). Jene Arbeit führte er bis in seine letzten Jahre fort; wegen nachlassender Sehkraft mußte er den Text in einem Monitor bis auf Handbreite vergrößern. Er bearbeitete auch kritische Ausgaben von khori- und selenginisch-burjatischen Chroniken (1935/40, 1936), des Mukaddimat al-Adab 1-2 (1938-39), der mongolischen Version des Lalitavistara (The Twelve Deeds of Buddha, 1967) und des Vajracchedika (The Diamond Sutra, 1971). Eines seiner Erstlingswerke war eine Übersicht über die finnisch-ugrischen Völker (1927, zusammen mit G. A. Starcev). Viele von seinen ursprünglich auf russisch geschriebenen wertvollen Monographien wurden später übersetzt. Seine hochinteressanten Lebenserinnerungen veröffentlichte er i.J. 1983 (Reminiscences, edited by Henry G. Schwarz. Studies on East Asia 16.). Seine Ausgaben der quadratschriftlichen Dokumente, des mongolischen Wörterbuches Muqaddimat al$A d a b$ und der Sprachmateriaiien einer Leidener Handschrift schätzte er selbst am höchsten ein.

Mit Finnland verband Poppe eine besondere Beziehung, was er häufig betonte. Es war ja unter anderem das Land, in dem er die Sommer seiner Kindheit verbracht hatte. Er konnte Parikkala 1960 wieder besuchen. In Helsinki traf er die Witwe und Tochter Ramstedts sowie finnische Kollegen. Später wiederholte er jene Besuche, wobei er von Professor Pentti Aalto mit Familie freundlichst unterstützt wurde. Nach dem Tod Poppes hielten die in Seattle ansässigen Finnen zu seinen Ehren eine Gedenkversammlung.

Die Wirkung des großen Gelehrten Nikolaus Poppe ist unübersehbar. Kaum ein Altaist (im weitesten Sinn) kann ohne seine Werke irgendetwas beginnen. Dadurch fühlt man sich als sein Schüler, so wie er selbst sich als Schüler Ramstedts fühlte, obwohl sie einander nur zweimal flüchtig begegnet waren. Menschliche Wärme, Ausgewogenheit und große Präzision sprechen aus seinen Werken, durch dic er noch lange Zeit unter uns leben bleiben wird.

HARRY HALÉN 